\title{
Quantum Mechanics of Moving Bound States
}

\author{
Y. S. Kim \\ Department of Physics, University of Maryland College Park, Maryland, USA \\ Email: yskim@umd.edu
}

How to cite this paper: Kim, Y.S. (2022) Quantum Mechanics of Moving Bound States. Journal of Modern Physics, 13, 138165.

https://doi.org/10.4236/jmp.2022.132012

Received: December 7, 2021

Accepted: February 14, 2022

Published: February 17, 2022

Copyright $\odot 2022$ by author(s) and Scientific Research Publishing Inc. This work is licensed under the Creative Commons Attribution International License (CC BY 4.0).

http://creativecommons.org/licenses/by/4.0/ (c) (i) Open Access

\begin{abstract}
The quantum mechanics of bound states with discrete energy levels is well understood. The quantum mechanics of scattering processes is also well understood. However, the quantum mechanics of moving bound states is still debatable. When it is at rest, the space-like separation between the constituent particles is the primary variable. When the bound state moves, this space-like separation picks up the time-like separation. The time-separation is not a measurable variable in the present form of quantum mechanics. The only way to deal with this un-observable variable is to treat it statistically. This leads to rise of the statistical variables such entropy and temperature. Paul A. M. Dirac made efforts to construct bound-state wave functions in Einstein's Lorentzcovariant world. In 1927, he noted that the c-number time-energy relation should be incorporated in the relativistic world. In 1945, he constructed fourdimensional oscillator wave functions with one time coordinate in addition to the three-dimensional space. In 1949, Dirac introduced the light-cone coordinate system for Lorentz transformations. It is then possible to integrate these contributions made by Dirac to construct the Lorentz-covariant harmonic oscillator wave functions. This oscillator system can explain the proton as a bound state of the quarks when it is at rest, and explain the Feynman's parton picture when it moves with a speed close to that of light. While the un-measurable time-like separation becomes equal to the space-like separation at this speed, the statistical variables become prominent. The entropy and the temperature of this covariant harmonic oscillator are calculated. It is shown that they rise rapidly as the proton speed approaches that of light.
\end{abstract}

\section{Keywords}

Bound States in Einstein's World, Bohr and Einstein on the Hydrogen Atom, Quark-Parton Puzzle, Lorentz Group

\section{Introduction}

Let us start with Figure 1. During the early years of the 20-th Century, Niels 
Bohr was worrying about the electron orbit of the hydrogen atom, while Albert Einstein was interested in how things appear to moving observers. Bohr's concern led to the present form of quantum mechanics where the hydrogen atom is a standing wave localized within a finite region. Einstein formulated his special theory of relativity based on the Lorentzian geometry of space and time applicable to Maxwell's theory of electromagnetism.

It is known that Bohr and Einstein met occasionally to discuss physics. However, there are no written records to indicate that they ever discussed how moving hydrogen atoms appear to a stationary observer. If they did not discuss this problem, it is understandable because there are no observable hydrogen atoms moving with relativistic speeds. Yet, this Bohr-Einstein issue defines an important problem in quantum mechanics. The bound state in quantum mechanics with discrete energy levels is well understood. However, how would those energy levels appear to moving observers? What will happen to the size of the bound state? Indeed, the Bohr-Einstein issue of the hydrogen atom leads to the problem of moving quantum bound states in Einstein's Lorentz-covariant world.

There are a number of key questions on the moving bound state. In the Lorentz-covariant world, the time variable is linearly mixed with the longitudinal coordinate. There is also the time-energy uncertainty relation. How is this relation mixed with Heisenberg's uncertainty for momentum and space? Paul A. M. Dirac raised these questions in 1927, and attempted to find a solution using harmonic oscillator wave functions in 1945. In addition, in 1949, he introduced the light-cone coordinate system for squeeze transformations in the two-dimensional space of the time and longitudinal coordinate.

The Bohr radius is a spatial separation between the proton and electron in the hydrogen atom. If this atom is boosted, this spatial separation picks up its timelike component. However, this time-like separation is not included in the present form of quantum mechanics. On the other hand, it is still possible to regard this time separation as an un-observable variable and treat it statistically, using the density matrix [1]-[11]. Then, there comes the question of entropy and temperature from this statistical treatment.
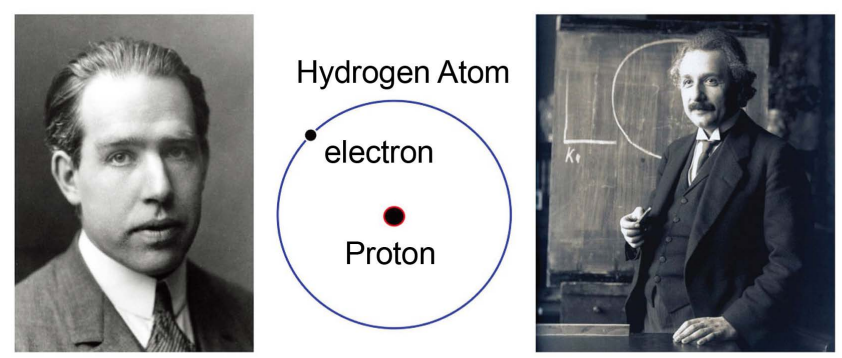

Figure 1. Niels Bohr and Albert Einstein with the hydrogen atom. One hundred years ago, Bohr was worrying about why the radius of the electron in the hydrogen atom orbit cannot be smaller than the finite value known today as the Bohr radius. Einstein was interested in how things appear to moving observers. Then, how would the hydrogen atom appear to moving observers? This question defines the subject of quantum mechanics of bound states in the Lorentz-covariant world. 
While there are no observable hydrogen atoms, these days, high-energy accelerator produce protons moving with speeds close to that of light. Furthermore, thanks to Gell-Mann's quark model [12], the proton was found to be a quantum bound state just like the hydrogen atom. Its constituents are the quarks. Since the proton and hydrogen atom share the same bound-state quantum mechanics, it is possible to study moving hydrogen atoms by looking at moving protons.

In 1969, Feynman observed that the ultra-fast proton appears like a collection of an infinite-number of free particles with a wide-spread momentum distribution [13] [14] [15]. Feynman called them partons. The question then is whether Gell-Mann's quarks and Feynman's partons are two different ways of observing the same entity. Indeed, the problem of moving hydrogen atom becomes the quark-parton puzzle. The Bohr-Einstein issue of moving hydrogen atom can be addressed in terms of the quark-parton puzzle of Gell-Mann and Feynman, as illustrated in Figure 2.

In this paper, we review first efforts made in the past to resolve this quark-parton issue [16] [17], using the Lorentz-covariant oscillator wave function. We then use the same wave function to study the problem arising from the un-observable time-separation variable.

Paul A. M. Dirac made his lifelong efforts to construct a localized wave function in Einstein's Lorentz-covariant world. For this purpose, Dirac published

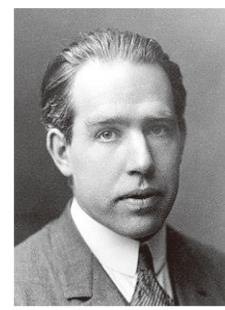

100 years ago, Bohr was worrying about the orbit of the hydrogen atom.

Einstein was interested in how things look to moving observers. Then how the hydrogen atom would look to moving observers? This was a metaphysical question for them.

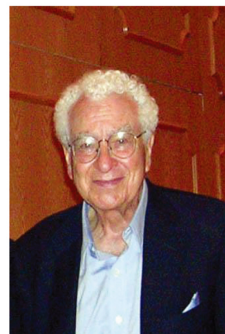

50 years ago, the proton became a bound state of the quarks sharing the same quantum mechanics as that for the hydrogen atom, according to Gell-Mann. If it moves with a speed close to that of light, the proton appears as a collection of partons, according to Feynman.

Question. Does the proton appear like a collection of Feynman's partons to a moving observer?

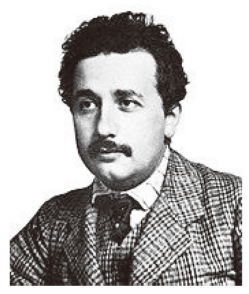

Photo of Gell-Mann by Y.S.Kim (2010), all others photos are from the public domain.

Figure 2. Bohr and Einstein, and then Gell-Mann and Feynman. Did Bohr and Einstein discuss how the hydrogen appears to moving observers? We do not know. After 1950, with particle accelerators, the physics world started producing protons with relativistic speeds. Furthermore, the proton became a quantum bound state of the quarks like the hydrogen atom. The problem of fast-moving hydrogen atoms became the problem of protons moving with relativistic speeds. How would the proton appear when it moves with a speed close to that of light? This quark-parton puzzle addresses the Bohr-Einstein issue of moving hydrogen atom. 
four important papers [18] [19] [20] [21]. By integrating the first three of these four papers, it is possible to construct the harmonic oscillator wave functions which can be Lorentz-transformed [22] [23] [24].

In order to carry out this integration, we need the mathematical instrument constructed by Eugene P. Wigner in his 1939 paper on the inhomogeneous Lorentz group [25]. In his paper, Wigner pointed out a particle in the Lorentzcovariant world has its four-momentum. In addition, this particle has internal space-time symmetries.

Thus in Section 2, we review the aspects of Wigner's paper applicable to the internal space-time symmetries in Einstein's Lorentz-covariant world.

In Section 3, we list the first three papers Dirac published from 1927 to 1945 [18] [19] [20], and integrate them. The result is a harmonic oscillator wave function which can be Lorentz-boosted. The time-separation variable plays a prominent role in this Lorentz-covariant wave function.

In Section 4, we examine Feynman's attempts to construct Lorentz-covariant oscillator wave function starting from a Lorentz-invariant wave equation [26]. Let us consider a hadron (bound state of the quarks) consisting of two quarks. This hadron has two space-time coordinate systems. One is for the hadron moving freely, and the other is for the motion of quarks inside the hadron. For the hadronic coordinate, the Klein-Gordon equation and it solutions are applicable. For the internal coordinate, we can use the harmonic oscillator wave functions constructed from the integration of Dirac's three papers discussed in Section 3.

In Section 5, this covariant harmonic oscillator is applied to the physics of hadrons. This wave function allows us to Lorentz-boost the hadron at rest to its speed very close to that of light. The hadron at rest is like a quantum bound state like the hydrogen atom according to Gell-Mann's quark model [12]. However, the same hadron appears like a collection of free massless particles called partons. This aspect is called Feynman's parton picture of the hadron [13] [14] [15]. The question then is whether the quarks and partons are two different ways of looking at the same entity. We resolve this issue using the Lorentz-covariant oscillator wave functions constructed in Sections 3 and 4. This Lorentz-covariant wave function depends on the time-separation variable which becomes more prominent as the hadron gains its speed.

In Section 6, it is noted that there is a time-separation variable between the quarks. This variable becomes more prominent when the hadron becomes faster. This time-separation is not a measurable dynamical variable in the present form of quantum mechanics. However, the density matrix tells us how to deal with this unobservable variable. It allows us to translate our inability to measure this variable into entropy and temperature. This problem was discussed in the literature [27] [28]. It is shown there that the hadron, when Lorentz boosted, experiences the rise in entropy and also the rise in temperature. It is possible to calculate them as functions of the hadronic speed using the density matrix. 


\section{Wigner's Little Groups for Internal Space-Time Symmetries}

In 1939, Eugene Paul Wigner published his paper entitled On unitary representations of the inhomogeneous Lorentz group [25]. In this paper, Wigner spells out the internal space-time symmetries of particles in the Lorentz-covariant world [22]. Let us consider a particle in this world. It has its four-momentum. If this particle is at rest, it has its rotational degree of freedom. If its spin is $1 / 2$, the symmetry group is $S U(2)$ like (locally isomorphic to) $O(3)$ (three-dimensional rotation group). If its spin is one, its symmetry group is $O(3)$. This aspect is well known.

Massless particles cannot be brought to their rest frames. According to Wigner [25], the little group for the massless particle is like $E(2)$ or the two-dimensional Euclidean group, with one rotational degree of freedom plus two translational degrees of freedom. The rotational degree of freedom can easily be identified with the helicity of the massless particle. However, the two translational degrees have a stormy history until 1987, when Kim and Wigner noted that the $E(2)$ group is like the cylindrical group where both the translations perform up-down translations on the cylindrical surface. This allows us to identify this up-down translation as with the gauge transformation [29].

In the Lorentz-covariant world, Einstein's momentum-energy relation is applicable to both massive and massless particles, as shown in Table 1 . When the massive particle is Lorentz-boosted, its energy-momentum becomes that of the massless particle when its speed becomes very close to that of light.

We are thus led to the question of whether there exists one little group which can be the $O(3)$-like group when the particle is at rest and the $E(2)$-like cylindrical group when the particle moves with the speed very close to that of light.

Let us outline the procedure in which the $O(3)$ symmetry becomes that of the cylindrical group. The three-dimensional rotation group is generated by

$$
L_{x}=-i\left(y \frac{\partial}{\partial z}-z \frac{\partial}{\partial y}\right), \quad L_{y}=-i\left(z \frac{\partial}{\partial x}-x \frac{\partial}{\partial z}\right), \quad L_{z}=-i\left(x \frac{\partial}{\partial y}-y \frac{\partial}{\partial x}\right),
$$

Table 1. Lorentz covariance of particles with internal space-time symmetries. The first row tells that Einstein's energy relation is applicable to both massive and massless particles. Likewise, the second row is Wigner's little groups. They are like $O(3)$ for massive particles, and are like $E(2)$ for massless particles, where $E(2)$ means the two-decisional Euclidean group which is isomorphic to the cylindrical group.

\begin{tabular}{cccc}
\hline & Massive, Slow & COVARIANCE & Massless, Fast \\
\hline Energy-Momentum & $E=p^{2} / 2 m$ & $E=\sqrt{(c p)^{2}+\left(m c^{2}\right)^{2}}$ & $E=c p$ \\
Internal & $S_{3}$ & Winstein's & \\
Space-time & $S_{1}, S_{2}$ & Little Groups & $S_{3}$ \\
Symmetry & & & Gauge \\
\end{tabular}


satisfying the commutation relations

$$
\left[L_{x}, L_{y}\right]=i L_{z}, \quad\left[L_{y}, L_{z}\right]=i L_{x}, \quad\left[L_{z}, L_{x}\right]=i L_{y} .
$$

Let us go to Figure 3. The circle in this figure illustrates the $O(3)$-like little group for the massive particle at rest.

If it is boosted along the z-direction, the $z$ coordinate picks up the time-like component, and the geometry is four-dimensional. While this geometry is described in detail in the 1987 paper of Kim and Wigner [29], we give here a simplified version.

When the particle is boosted along the $z$ direction, the $z$ component of this circle becomes expanded. If the speed becomes close to that of light, the sphere becomes a cylinder, as indicated in Figure 3.

On the surface of this cylinder, there are no variations of the $x$ and $y$ components, and thus

$$
L_{x} \rightarrow P_{y}=-i\left(y \frac{\partial}{\partial z}\right), \quad L_{y} \rightarrow-P_{x}=i\left(x \frac{\partial}{\partial z}\right),
$$

and $L_{z}$ remains unchanged. Both $P_{x}$ and $P_{y}$ generate translations along the $z$ direction. These new operators satisfy the commutation relations

$$
\left[P_{x}, P_{y}\right]=0, \quad\left[L_{z}, P_{x}\right]=i P_{y}, \quad\left[L_{z}, L_{y}\right]=-i P_{x} .
$$

It is appropriate to call the group generated by these three operators the $c y$ lindrical group.

This set of commutation relation identical with that for $E(2)$ or the twodimensional Euclidean group with $L_{z}$ as the generator of rotations and $P_{x}$ and $P_{y}$ as the generators of translations along the $x$ and $y$ directions respectively. The translation generators take the form

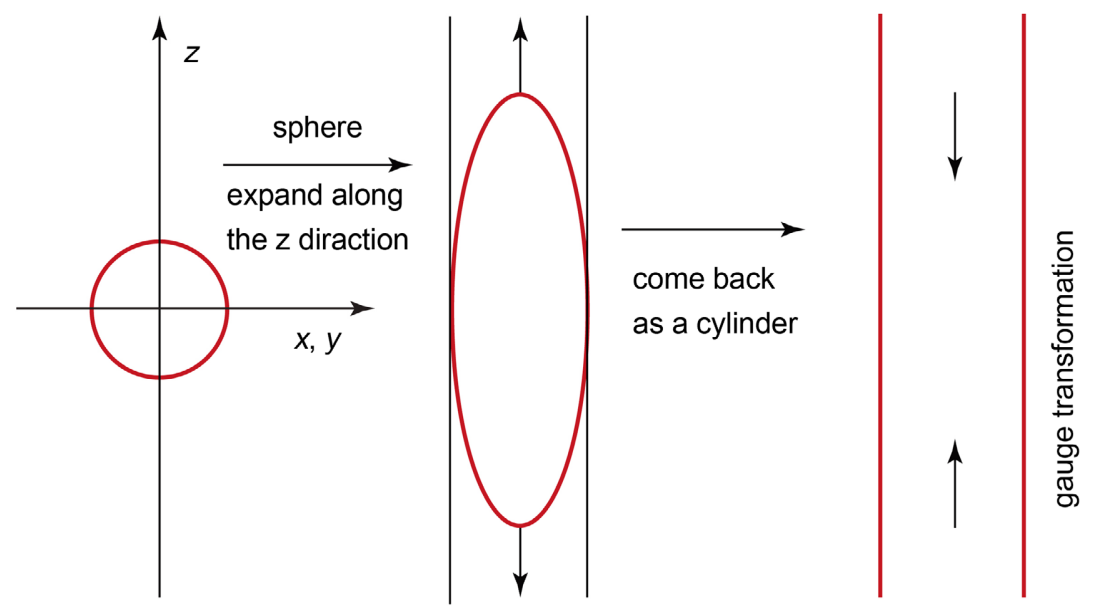

Figure 3. Lorentz covariance of the internal space-time symmetry. The symmetry is like $O(3)$ or a sphere when the particle is at rest. This sphere becomes elongated when the particle gains speed along the $z$ direction. It becomes a cylinder when the speed approaches the speed of light. The rotational degree of freedom of this cylinder corresponds to the helicity of the massless particle, and up-down translation leads to the gauge degree of freedom. 


$$
P_{x}=-i \frac{\partial}{\partial x}, \quad P_{y}=-i \frac{\partial}{\partial y} .
$$

Thus, the cylindrical group is like (locally isomorphic to) the $E(2)$ group. In Wigner's original paper, the little group for massless particles is like the $E(2)$ group. The $E(2)$ group can now replaced with the cylindrical group [29] [30].

Let us go back to Figure 3. We would expect the sphere, when Lorentz-boosted, becomes contracted like a pancake according to Einstein's space contraction. However, this figure shows the opposite effect. The Lorentzian geometry is fourdimensional and the time-like direction should also be included. In this geometry, the Lorentz boost leads to both pancake-like contraction and football-like elongation. The contraction produces the $E(2)$ geometry and the elongation produces the cylindrical geometry as shown in Figure 3. The cylindrical geometry leads to the correct interpretation of the internal space-time symmetry of massless particles.

\section{Dirac's Efforts to Construct Relativistic Quantum Mechanics}

Paul A. M. Dirac made his lifelong effort to formulate quantum mechanics consistent with Einstein's special relativity. The Dirac equation of electrons and positrons is a case in point. This equation is well known.

In addition, he made efforts to formulate a mathematical device to deal localized quantum distributions, such as the hydrogen atom, in Einstein's Lorentzcovariant world. For this purpose, he published the following four papers.

1) In 1927, Dirac pointed out that the time-energy uncertainty should be considered if the system is to be Lorentz-covariant [18].

2) In 1945, Dirac said the Gaussian form could serve as a representation of the Lorentz group [19].

3) In 1949, when Dirac introduced both his instant form of quantum mechanics and his light-cone coordinate system [20], he clearly stated that finding a representation of the inhomogeneous Lorentz group was the task of Lorentzcovariant quantum mechanics.

4) In 1963, Dirac used the symmetry of two coupled oscillators to construct the $O(3,2)$ de Sitter group, namely the Lorentz group applicable to the threedimensional $(x, y, z)$ space plus two time variables [21]. This paper serves as a prelude to the synthesis of quantum mechanics and special relativity [24] [31] [32].

Dirac's papers are poetic, mathematically transparent, and easy to understand. This does not necessarily mean that there is nothing to add to his papers. His papers do not have figures. Thus it is profitable to translate his poems into figures and illustrations. Each of the above four papers is independent. Thus it is profitable to connect his 1945 paper to his early paper of 1927, and his 1949 paper to his earlier papers of 1927 and 1945. Furthermore, Eugene Wigner was his brother-in-law. Wigner published an important paper is 1939 providing the 
mathematical tool for Dirac's problems, but he never used Wigner's mathematics in any meaningful ways.

Let us consider what we can add to his papers in order to construct quantum mechanics valid in Einstein's Lorentz-covariant world. We are particularly interested in how to Lorentz-boost localized wave functions.

\subsection{Dirac's C-Number Time-Energy Uncertainty Relation}

In 1972 [33], Eugene Paul Wigner drew attention to the fact that time-energy uncertainty relation, known from the transition time and line broadening in atomic spectroscopy, existed before 1927 when Heisenberg formulated his uncertainty principle.

In 1927 [18], Dirac studied the uncertainty relation which was applicable to the time and energy variables. When the uncertainty relation was formulated by Heisenberg for the position and momentum variables, Dirac considered the possibility of whether a Lorentz-covariant uncertainty relation could be formulated with these two uncertainty relations [18].

Dirac then noted that the time variable is a c-number and thus there are no excitations along the time-like direction. However, there are excitations along the space-like longitudinal direction starting from the position-momentum uncertainty. Since the space and time coordinates are mixed up for moving observers, Dirac wondered how this space-time asymmetry could be made consistent with Lorentz covariance. This was indeed a major difficulty for him.

However this difficulty does not exist. Wigner's little group for massive particles at rest is the three-dimensional rotation group, without time-dependence. The concept of the little group did not exist in 1927.

\subsection{Dirac's Four-Dimensional Oscillators}

Since the language of special relativity is the Lorentz group, and harmonic oscillators provide a starting point for the present form of quantum mechanics, Dirac considered the possibility of using harmonic oscillator wave functions to construct representations of the Lorentz group [19].

Thus in his 1945 paper [19], Dirac considers the Gaussian form

$$
\exp \left(-\frac{1}{2}\left[x^{2}+y^{2}+z^{2}+t^{2}\right]\right) \text {. }
$$

The $x$ and $y$ variables can be dropped from this expression, as we are considering a Lorentz boost only along the $z$ direction. We can thus write the above equation as:

$$
\exp \left(-\frac{1}{2}\left[z^{2}+t^{2}\right]\right)
$$

Since $\left(z^{2}-t^{2}\right)$ is a Lorentz-invariant quantity, this expression may seem strange for those who believe in Lorentz invariance [26], but it is normalizable in the $t$ variable, and accommodates the time-energy uncertainty relation. This noninvariant form will change when it is boosted along the $z$ direction. 
However, there are no excitations along the time-like direction. This space-time asymmetry was noted in Dirac's own paper of 1927 [18]. This asymmetry problem was resolved by Wigner's $O(3)$-like little group for massive particles discussed in Section 2. Without time-like excitations, the oscillator wave function should take the form

$$
\psi^{n}(z, t)=\left(\frac{1}{\pi n ! 2^{n}}\right)^{1 / 2} H_{n}(z) \exp \left(-\left[\frac{z^{2}+t^{2}}{2}\right]\right),
$$

for the n-th excited state, where $H_{n}(z)$ is the Hermite polynomial for excitations along the $z$ direction. This expression does not contain the Hermite polynomial in the $t$ variable.

Since the localization of this wave function is dominated by the Gaussian form, let us concentrate our efforts on the ground state. For this ground state, this wave function become

$$
\psi(z, t)=\left(\frac{1}{\pi}\right)^{1 / 2} \exp \left(-\left[\frac{z^{2}+t^{2}}{2}\right]\right) .
$$

This corresponds to the circular distribution in Figure 4. Since the form is not Lorentz-invariant, the circle in Figure 4 will appear differently to moving observers. This question was addressed in Dirac's 1949 paper [20].

Bohr

Quantum Mechanics

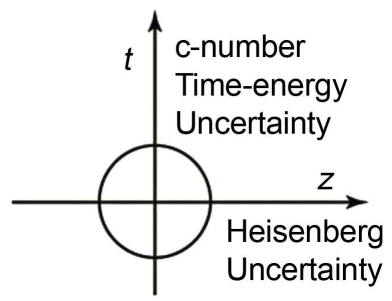

Einstein

\section{Lorentz Covariance}

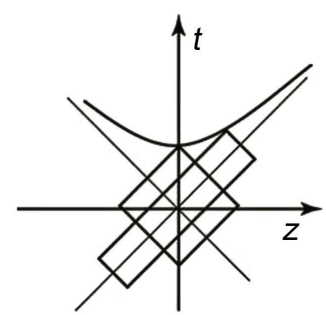

Synthesis of Bohr and Einstein

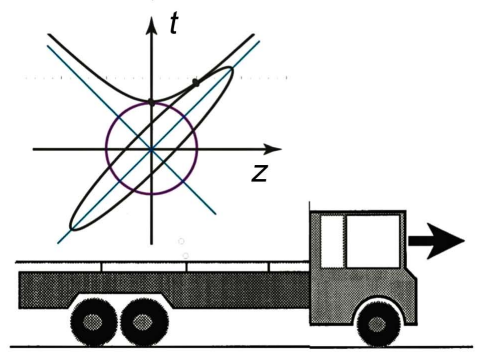

Figure 4. Integration of Dirac's three papers [18] [19] [20]. In 1927, Dirac noted there exists the time-energy uncertainty, in addition to Heisenberg's position-momentum relation [18]. He attempted to combine them with a Gaussian form in 1945 [19]. In 1949, Dirac noted that the Lorentz boost squeezes space-time along the light cones. This allows us to synthesize the circle and rectangle to an ellipse for the moving oscillator. This figure provides the resolution to the Bohr-Einstein issue of the moving hydrogen atom. The remaining question is whether we can observe this effect in laboratories. 


\subsection{Forms of Relativistic Dynamics}

In 1949, the Reviews of Modern Physics celebrated Einstein's 70th birthday by publishing a special issue. This issue included Dirac's paper entitled Forms of Relativistic Dynamics [20]. There, Dirac introduced his light-cone coordinate system. In this system a Lorentz boost is seen to be a squeeze transformation, where one light-cone axis expands while the other contracts in such a way that their product remains invariant as shown in Figure 4.

Also in this 1949 paper [20], Dirac introduced his instant form of relativistic quantum mechanics. This has the condition

$$
x_{0} \approx 0 \text {. }
$$

What did his approximate equality mean? We can interpret this as his c-number nature of the time-energy uncertainty relation which he discussed in his 1927 paper [18]. In the language of harmonic oscillators [19], there are no excited states along the time axis, as is shown in Equation (8).

In the same 1949 paper, Dirac introduced the light-cone coordinate system. Starting from the formula's for the Lorentz boost along the $z$ direction:

$$
\left(\begin{array}{l}
z^{\prime} \\
t^{\prime}
\end{array}\right)=\left(\begin{array}{cc}
\cosh (\eta) & \sinh (\eta) \\
\sinh (\eta) & \cosh (\eta)
\end{array}\right)\left(\begin{array}{l}
z \\
t
\end{array}\right)
$$

Dirac defined his light-cone variables as [20]

$$
u=\frac{z+t}{\sqrt{2}}, \quad v=\frac{z-t}{\sqrt{2}} .
$$

Then the Lorentz boost of Equation (11) becomes diagonal:

$$
\left(\begin{array}{c}
u^{\prime} \\
v^{\prime}
\end{array}\right)=\left(\begin{array}{cc}
\mathrm{e}^{\eta} & 0 \\
0 & \mathrm{e}^{-\eta}
\end{array}\right)\left(\begin{array}{l}
u \\
v
\end{array}\right)
$$

It is then apparent that $u$ variable becomes expanded, but the $v$ variable becomes contracted. This aspect was illustrated also in Figure 4. The product then becomes

$$
u v=u^{\prime} v^{\prime}=\frac{1}{2}(z+t)(z-t)=\frac{1}{2}\left(z^{2}-t^{2}\right)
$$

which remains invariant. The Lorentz boost is therefore a squeeze transformation, and the Gaussian form of Equation (9) is transformed to.

$$
\psi_{\eta}(z, t)=\left(\frac{1}{\pi}\right)^{1 / 2} \exp \left(-\frac{1}{4}\left[\mathrm{e}^{-2 \eta}(z+t)^{2}+\mathrm{e}^{2 \eta}(z-t)^{2}\right]\right) .
$$

This is of course the elliptic distribution as noted in Figure 4.

In addition, in his 1949 paper [20], Dirac stated that the task of constructing relativistic quantum mechanics is that of constructing a representation of the inhomogeneous Lorentz group, which is also known as the Poincaré group [22]. This group has ten generators for three rotations, three Lorentz boosts, and four space-time translations.

It is well known that the present form of quantum field theory based on the 
scattering matrix is a representation of the Poincare group. The question is whether it is possible to formulate the bound state problem as a representation of the same group.

It is now clear that Dirac was interested in using harmonic oscillators to construct a representation of the inhomogeneous Lorentz group [22]. Figure 5 indicates that the integration of Dirac's first three papers [18] [19] [20] lead to a representation of Wigner's $O(3)$-like little group for the massive particle. Since it is well known that quantum field theory is a representation of the inhomogeneous Lorentz group, both the field theory and the oscillator formalism are two different representations of the same inhomogeneous Lorentz group satisfying Dirac's requirement [20].

\subsection{Dirac's Two Oscillators}

In 1963, Dirac started with two harmonic oscillators, and he ended up with ten generators [21]. These generators satisfy the closed set of commentators for the $O(3,2)$ group, namely the Lorentz group applicable to three space-like coordinates and two time-like coordinates. This group has ten generators, like the Poincaré group. Like the Poincaré group, it has the subgroup $O(3,1)$ Lorentz group when we consider only one of the two time coordinates. There are four generators involving the second time coordinates, namely three boost generators with respect to three space coordinates and one rotation generator with respect to the first time variable.

The harmonic oscillator is the language of quantum mechanics, while the group $O(3,2)$ is the language of Lorentz transformations. Thus, we are led to the question of deriving special relativity from quantum mechanics.

According to Dirac [20], the task of constructing relativistic quantum mechanics is that of constructing a representation of the inhomogeneous Lorentz

\begin{tabular}{|c|c|c|}
\hline $\begin{array}{l}\text { Comets } \\
\text { Scattering }\end{array}$ & $\begin{array}{c}\text { Planets } \\
\text { Bound States }\end{array}$ & \multirow{4}{*}{ Galilei } \\
\hline \multicolumn{2}{|c|}{ Newton } & \\
\hline Rutherford & Bohr & \\
\hline \multicolumn{2}{|c|}{ Heisenberg and Schrödinger } & \\
\hline $\begin{array}{l}\text { Feynman } \\
\text { Diagrams }\end{array}$ & $\begin{array}{c}\text { Dirac } \\
(1927,45,49)\end{array}$ & \multirow{2}{*}{ Einstein } \\
\hline \multicolumn{2}{|c|}{ Inhomgeneous Lorentz Group } & \\
\hline Heisenb & Einstein. Po & ble? \\
\hline
\end{tabular}

Integration of Dirac

$(1927,1945,1949)$

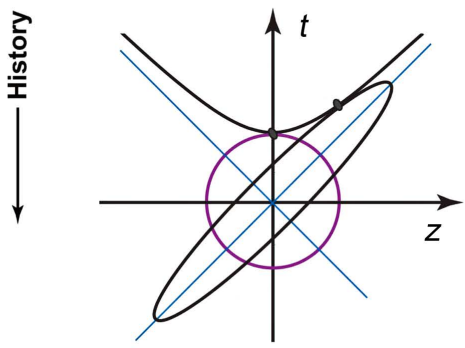

Figure 5. History of physics as a series of synthesis. Newton synthesized scattering and bound states with his differential equation. Schrödinger and Heisenberg synthesized the Rutherford scattering and the Bohr atom. There then comes Einstein's world. Quantum field theory is for scattering problem while the covariant harmonic oscillator is for bound states. They both are representations of the inhomogeneous Lorentz group. Next, are quantum mechanics and special relativity are derivable from the same basket of equations? This question is addressed in the literature [24] [31] [32]. 
group with ten generators, as specified in Figure 5. Dirac's $O(3,2)$ group also has ten generators. It is thus a challenge to see whether this $O(3,2)$ group can be converted into the inhomogeneous Lorentz group. This question was discussed in detail in the literature [24] [31] [32].

\section{Scattering and Bound States}

In Section 3, we studied the quantum bound state in the Lorentz-covariant world using harmonic oscillator wave functions. This wave function is a representation of Wigner's little group, which is a subgroup of Dirac's inhomogeneous Lorentz group.

For free particles in the covariant world, we use the Klein-Gordon equation. If the particle has a space-time extension, it is possible to use harmonic oscillators as we did in Section 3. The question then is whether it is possible to write an equation for both. Indeed, this problem was recognized by Feynman, Kislinger, and Revndal in 1971 [26].

Let us start with two quarks. We are quite familiar with the Klein-Gordon equation for a free particle in the Lorentz-covariant world. We shall use the fourvector notations

$$
x_{\mu}=(x, y, z, t), \quad \text { and } \quad x_{\mu}^{2}=x^{2}+y^{2}+z^{2}-t^{2} .
$$

Then the Klein-Gordon equation becomes

$$
\left(-\left[\frac{\partial}{\partial x_{\mu}}\right]^{2}+m^{2}\right) \phi(x)=0
$$

The solution of this equation takes the familiar form

$$
\exp \left[ \pm i\left(p_{1} x+p_{2} y+p_{3} z \pm E t\right)\right]
$$

with $E=\sqrt{p_{1}^{2}+p_{2}^{2}+p_{3}^{2}+m^{2}}$.

In 1971, Feynman et al. considered two particles $a$ and $b$ bound together by a harmonic oscillator potential, and wrote down the equation [26]

$$
\left\{-\left[\frac{\partial}{\partial x_{a \mu}}\right]^{2}-\left[\frac{\partial}{\partial x_{b \mu}}\right]^{2}+\left(x_{a \mu}-x_{b \mu}\right)^{2}+m_{a}^{2}+m_{b}^{2}\right\} \phi\left(x_{a \mu}, x_{b \mu}\right)=0 .
$$

The bound state of these two particles is one hadron. The constituent particles are called quarks. We can then define the four-coordinate vector of the hadron as

$$
X=\frac{1}{2}\left(x_{a}+x_{b}\right),
$$

and the space-time separation four-vector between the quarks as

$$
x=\frac{1}{2 \sqrt{2}}\left(x_{a}-x_{b}\right) .
$$

Then Equation (19) becomes 


$$
\left\{-\left[\frac{\partial}{\partial X_{\mu}}\right]^{2}+m_{0}^{2}+\left(-\left[\frac{\partial}{\partial x_{\mu}}\right]^{2}+x_{\mu}^{2}\right)\right\} \phi(X, x)=0 .
$$

This differential equation can then be separated into

$$
\left(-\left[\frac{\partial}{\partial X_{\mu}}\right]^{2}+m_{0}^{2}\right) \phi(X, x)=-\left(-\left[\frac{\partial}{\partial x_{\mu}}\right]^{2}+x_{\mu}^{2}\right) \phi(X, x),
$$

with

$$
\phi(X, x)=f(X) \psi(x),
$$

where $f(X)$ and $\psi(x)$ satisfy their own equations:

$$
\left(-\left[\frac{\partial}{\partial X_{\mu}}\right]^{2}+m_{a}^{2}+m_{b}^{2}+\lambda\right) f(X)=0
$$

and

$$
\frac{1}{2}\left(-\left[\frac{\partial}{\partial x_{\mu}}\right]^{2}+x_{\mu}^{2}\right) \psi(x)=\lambda \psi(x) .
$$

Here, the wave function takes the form

$$
\phi(X, x)=\psi(x) \exp \left[ \pm i\left(P_{x} X+P_{y} Y+P_{z} Z \pm E T\right)\right],
$$

where $P_{x}, P_{y}, P_{z}$ are for the hadronic momentum, and

$$
E^{2}=P_{x}^{2}+P_{y}^{2}+P_{z}^{2}+M^{2} \text {, with } M^{2}=m_{a}^{2}+m_{b}^{2}+\lambda .
$$

Here the hadronic mass $M$ is determined by the parameter $\lambda$, which is the eigenvalue of the differential equation for $\psi(x)$ given in Equation (26).

Considering Feynman diagrams based on the S-matrix formalism, quantum field theory has been quite successful. It is, however, only useful for physical processes where, after interaction, one set of free particles becomes another set of free particles. The questions of localized probability distributions and their Lorentz covariance is not addressed by the present form of quantum field theory. In order to tackle this problem and address these questions, Feynman et al. suggested harmonic oscillators [26]. In Figure 6, we illustrate this idea.

However, for their wave function $\psi(x)$, Feynman et al. uses a Lorentz-invariant exponential form

$$
\exp \left(-\frac{1}{2}\left[x^{2}+y^{2}+z^{2}-t^{2}\right]\right)
$$

This wave function increases as $t$ becomes large. This is not an acceptable wave function. They overlooked the normalizable exponential form given by Dirac in Equation (6). They even overlooked the same normalizable in the paper of Fujimura et al. [34] which was quoted in their own paper.

Thus, we are fully justified in replacing the meaningless Gaussian form of Equation (29) with the Gaussian form developed in Section 3. 


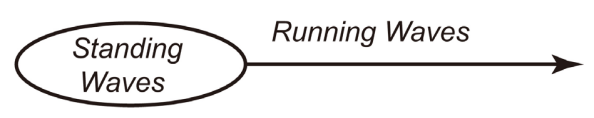

(a)

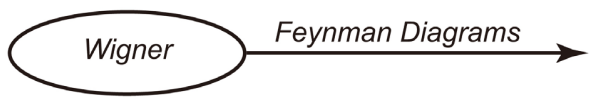

(b)

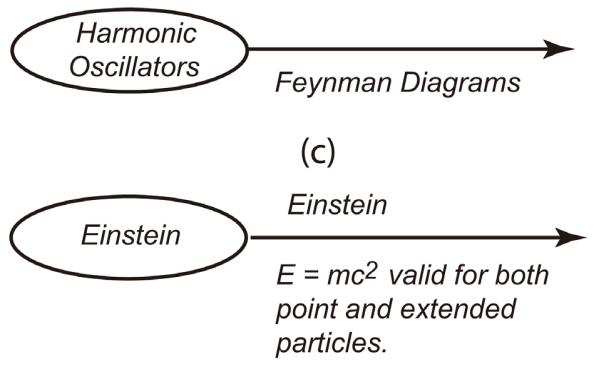

(d)

Figure 6. Scattering and bound states as a single representation of the inhomogeneous Lorentz group. In an effort to combine quantum mechanics with special relativity, Feynman gave us this road-map. In (a), we start with a running wave and standing wave. In (b), running waves are for Feynman diagrams, and standing waves are representations of Wigner's little group. In (c), we can use harmonic oscillator wave functions for standing waves. If the oscillator wave functions are Lorentz-covariant, Einstein's Lorentz covariance is valid for the entire system, as specified in (d).

\section{Lorentz-Covariant Quark Model}

Early successes in the quark model include the calculation of the ratio of the neutron and proton magnetic moments [35], and the hadronic mass spectra [26] [36]. These are based on hadrons at rest. We are interested in this paper how the hadrons in the quark model appear to observers in different Lorentz frames.

These days, modern particle accelerators routinely produce protons moving with speeds very close to that of light. Therefore, the question is whether the covariant wave function developed in Section 4 can explain the observed phenomena associated with those protons moving with relativistic speed.

The idea that the proton or neutron has a space-time extension had been developed long before Gell-Mann's proposal for the quark model [12]. Yukawa [37] developed this idea as early as 1953 , and his idea was followed up by Markov [38].

Hofstadter [39] [40], by using electron-proton scattering to measure the charge distribution inside the proton, made the first experimental discovery of the nonzero size of the proton. If the proton were a point particle, the scattering amplitude would just be a Rutherford formula. However, Hofstadter found a tangible departure from this formula which can only be explained by a spread-out charge distribution inside the proton.

Indeed, the first success of the Lorentz-covariant oscillator was demonstrated in the calculation of the Hofstadter effect. Using this wave function, Markov made his calculation in 1956 even before Gell-Mann formulated his quark model 
in 1964 [38]. After the quark model, many authors made their calculations of the Hofstadter effect using the same wave function [34] [41] [42] [43] [44] [45]. These papers amply demonstrate the elliptic deformation of the Gaussian distribution shown in Figure 4 and Figure 5.

Next, we are facing a more fundamental question. Let us go back to Figure 2. The quark model and its Lorentz-covariant wave function allow us to address the issue of the quark-parton puzzle, and thus the Bohr-Einstein issue of how the hydrogen atom appears to moving observers.

\subsection{Feynman's Parton Picture}

As we did in Sections 3 and 4, we continue using the Gaussian form for the wave function of the proton. If the proton is at rest, the $z$ and $t$ variables are separable, and the time-separation can be ignored, as we do in non-relativistic quantum mechanics. If the proton moves with a relativistic speed, the wave function is squeezed as described in Figure 4 and Figure 5. If the speed reaches that of light, the wave function becomes concentrated along positive light cone with $t=z$. The question then is whether this property can explain the parton picture of Feynman when a proton moves with a speed close to that of light.

It was Feynman who, in 1969, observed that a fast-moving proton can be regarded as a collection of many partons. The properties of these partons appear to be quite different from those of the quarks [13] [14] [15]. For example, while the number of quarks inside a static proton is three, the number of partons appears to be infinite in a rapidly moving proton. The following systematic observations were made by Feynman:

1) When protons move with velocity close to that of light, the parton picture is valid.

2) Partons behave as free independent particles while the interaction time between the quarks becomes dilated.

3) Partons have a widespread distribution of momentum as the proton moves quickly.

4) There seems to be an infinite number of partons or a number much larger than that of quarks.

The question is whether the Lorentz-squeezed wave function described in Figure 4 can explain all of these peculiarities.

Each of the above phenomena appears as a paradox, when the proton is believed to be a bound state of the quarks. This is especially true of (b) and (c) together. How can a free particle have a wide-spread momentum distribution.

To resolve this paradox, we construct the momentum-energy wave function corresponding to Equation (15). We can construct two independent four-momentum variables [26] if the quarks have the four-momenta $p_{a}$ and $p_{b}$.

$$
P=p_{a}+p_{b}, \quad q=\sqrt{2}\left(p_{a}-p_{b}\right) .
$$

Since $P$ is the total four-momentum, it is the four-momentum of the proton. The four-momentum separation between the quarks is measured by $q$. We can 
then write the light-cone variables as

$$
q_{+}=\frac{q_{0}+q_{z}}{\sqrt{2}}, \quad q_{-}=\frac{q_{0}-q_{z}}{\sqrt{2}} .
$$

This results in the ground-state momentum-energy wave function

$$
\phi_{\eta}\left(q_{z}, q_{0}\right)=\left(\frac{1}{\pi}\right)^{1 / 2} \exp \left\{-\frac{1}{2}\left[\mathrm{e}^{-2 \eta} q_{+}^{2}+\mathrm{e}^{2 \eta} q_{-}^{2}\right]\right\} .
$$

Since the harmonic oscillator is being used here, the momentum-energy wave function has the mathematical form identical to that of the space-time wave function of Equation (15). These wave functions have the same Lorentz squeeze properties [16] [17]. These Lorentz-squeeze properties are illustrated in Figure 7.

From this figure, we can see that both wave functions behave like those for the static bound state of quarks when the proton is at rest with $\eta=0$. However, as $\eta$ increases, the wave functions become concentrated along their respective positive light-cone axes. This means that the quarks become like massless particles with wide space and momentum distributions. This is the property of Feynman's parton picture [13] [14].

Another puzzle is that quarks are coherent when the proton is at rest but the partons appear as incoherent particles. Does this mean that the Lorentz boost destroys coherence? Obviously, the answer to this question is NO.

When the proton is boosted, its matter becomes squeezed, as shown in Figure 7. The result is that the wave function for the proton becomes concentrated in the elliptic region along the positive light-cone axis, which is expanded in length by $\exp (\eta)$. As a consequence, the minor axis is contracted by $\exp (-\eta)$.

\section{Lorentz-squeezed Hadron}
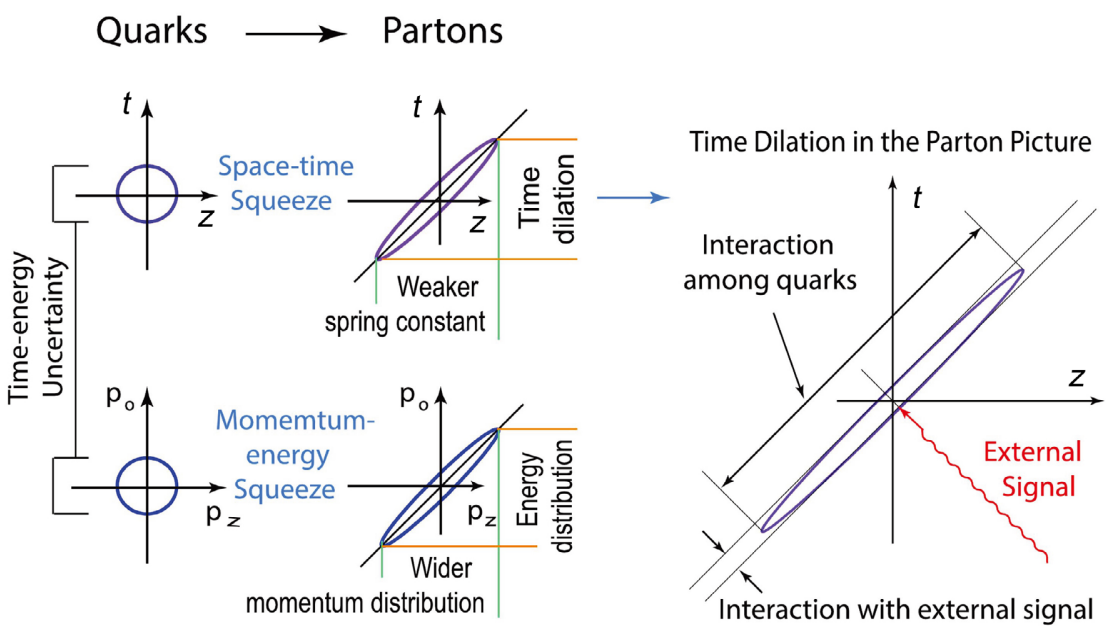

Figure 7. Lorentz-squeezed wave functions in space-time and in momentum-energy variables. Both wave functions become concentrated along their respective positive lightcone axes as the speed of the proton approaches that of light. All the peculiarities of Feynman's parton picture are presented in these light-cone concentrations. 
Thus, the interaction time for the quarks among themselves becomes dilated. Thus, the quarks appear to be free particles to external signals. As the ellipse becomes more squeezed, the quarks become light-like massless particles, as illustrated in Figure 8.

As indicated also in Figure 7, the probing signal is moving in the direction opposite to the direction of the proton, it travels along the negative light-cone axis with $t=-z$. As the proton contracts along this negative light-cone axis, the interaction time decreases by $\exp (-\eta)$. Then the ratio of the interaction time to the oscillator period becomes $\exp (-2 \eta)$. Each proton, produced by the Fermilab accelerator used to have an energy of $900 \mathrm{GeV}$. This then means that the ratio is $10^{-6}$. Because this is such small number, the external signal cannot sense the interaction of the quarks among themselves. The quarks, appearing like partons, are free independent particles as also observed by Feynman [13] [14].

The momentum distribution becomes wide spread, also as is indicated in Figure 7. As it becomes concentrated along the positive light-cone axis, the quarks become light-like massless particles. As in the case of the Black-body radiation, the number of particles is infinite with a continuos momentum (thus energy) distribution, also as noted by Feynman [13] [14].

This resolution of the quark-parton puzzle is tabulated in Table 2 along with Einstein's energy-momentum relation, and Wigner's little group for internal space-time symmetries. Indeed, the quarks and partons are two different way of looking at the same entity in Einstein's Lorentz-covariant world.

\subsection{Lorentz-Invariant Uncertainty Products}

In the harmonic oscillator regime, the energy-momentum wave functions take the same mathematical form, and the uncertainty relation in terms of the uncertainty products is well understood. However, in the present case, the oscillator wave functions are deformed when Lorentz-boosted, as shown in Figure 7.

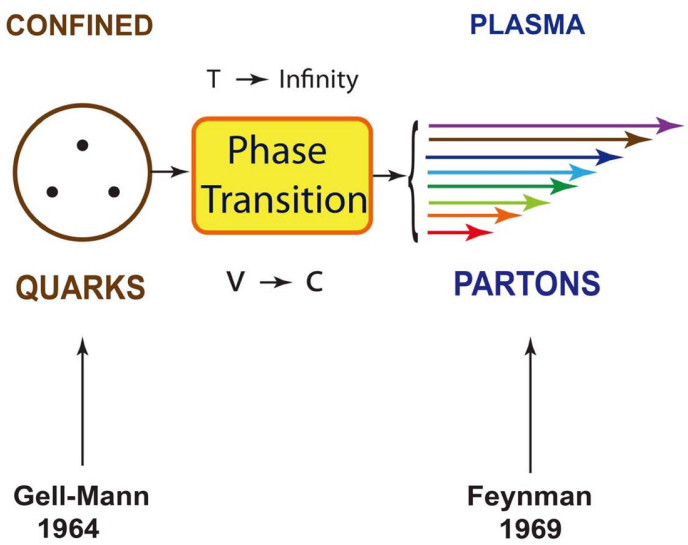

Figure 8. The effect of the Lorentz squeezes appearing in the real world. The Lorentzsqueezed wave functions shown in Figure 7 appear in the world as Feynman's parton picture in this figure. There are infinite-number of massless partons, with wide-spread momentum distribution, as in the case of photons in the black-body radiation. 
Table 2. Lorentz covariance of hadrons. The little group of Wigner unifies the internal space-time symmetries for massive and massless particles. This issue was addressed earlier in Table 1. Wigner's little groups allow us to give a unified picture for particles with internal space-time structures such as Gell-Mann's quark model and Feynman's parton picture.

\begin{tabular}{cccc}
\hline & Massive, Slow & COVARIANCE & Massless, Fast \\
\hline Energy-Momentum & $E=p^{2} / 2 m$ & $E=\sqrt{(c p)^{2}+\left(m c^{2}\right)^{2}}$ & $E=c p$ \\
Internal & $S_{3}$ & Wigner's & $S_{3}$ \\
Space-time & $S_{1}, S_{2}$ & Little Groups & $\begin{array}{c}\text { Gauge } \\
\text { Symmetry }\end{array}$ \\
Relativistic & & Integration & \\
Extended & Quark Model & of Dirac's papers \\
Particles & & 1927, 1945, 1949 & \\
\hline
\end{tabular}

According to this figure, both the space-time and momentum-energy wave functions become spread along their longitudinal directions. Does this mean that the Lorentz boost increases the uncertainty?

In order to address this question, let us write the momentum-energy wave function as a Fourier transformation of the space-time wave function:

$$
\phi\left(q_{z}, q_{0}\right)=\frac{1}{2 \pi} \int \psi(z, t) \exp \left\{i\left(q_{z} z-q_{0} t\right)\right\} \mathrm{d} t \mathrm{~d} z .
$$

The transverse $x$ and $y$ components are not included in this expression. The exponent of this expression can be written as

$$
q_{z} z-q_{0} t=q_{+} v+q_{-} u,
$$

with

$$
q_{ \pm}=\frac{1}{\sqrt{2}}\left(q_{z} \pm q_{0}\right) .
$$

In terms of these variables, the Fourier integral takes the form

$$
\frac{1}{2 \pi} \int \psi(z, t) \exp \left\{i\left(q_{+} v+q_{-} u\right)\right\} \mathrm{d} t \mathrm{~d} z .
$$

In this case, the variable $q_{+}$is conjugate to $v$, and $q_{-}$is to $u$. Let us go back to Figure 7. The major (minor) axis of the space-time ellipse is conjugate to the minor (major) axis of the momentum-energy ellipse. Thus the uncertainty products

$$
\left\langle u^{2}\right\rangle\left\langle q_{-}^{2}\right\rangle \text { and }\left\langle v^{2}\right\rangle\left\langle q_{+}^{2}\right\rangle
$$

remain invariant under the Lorentz boost.

\section{Entropy and Temperature of Moving Hadrons}

The entropy is a measure of our ignorance and is computed from the density matrix [1]-[11]. The density matrix is needed when the experimental procedure 
does not analyze all relevant variables to the maximum extent consistent with quantum mechanics. The purpose of the present section to discuss a concrete example of the entropy arising from our ignorance in relativistic quantum mechanics formulated in Sections 3 and 4.

Let us consider a bound state of two particles, or the hadron consisting of two quarks bound together by a harmonic oscillator potential. Then there is a Bohr-like radius measuring the space-like separation between the quarks. There is also the time-like separation in the Lorentz-covariant regime. If the hadron is at rest, the time dependence is purely Gaussian with no excitations. Thus, this un-observable variable can be integrated out without affecting the space-like separation.

If the hadron moves along the $z$ direction, this time-separation variable becomes more prominent, but there are no ways to measure this variable in the present form of quantum mechanics. We thus have to regard this variable as un-measurable variable, and treat it statistically.

As in the case of Section 4, let us consider a hadron consisting of two quarks. If the space-time position of two quarks are specified by $x_{a}$ and $x_{b}$ respectively, the system can be described by the variables

$$
X=\frac{x_{a}+x_{b}}{2} \text {, and } x=\frac{x_{a}-x_{b}}{2 \sqrt{2}} .
$$

The four-vector $X$ specifies where the hadron is located in space and time, while the variable $x$ measures the space-time separation between the quarks. In the convention of Feynman et al [26], the internal motion of the quarks bound by a harmonic oscillator potential can be described by the Lorentz-invariant equation

$$
\frac{1}{2}\left\{\left[-\frac{\partial^{2}}{\partial z^{2}}+z^{2}\right]-\left[-\frac{\partial^{2}}{\partial t^{2}}+t^{2}\right]\right\} \psi(z, t)=\lambda \psi(z, t) .
$$

For simplicity, we do not consider the transverse coordinates $x$ and $y$.

It is possible to construct a representation of Dirac's inhomogeneous Lorentz group [22] from the solutions of the differential equation of Equation (39). If the hadron is at rest, the solution should take the form of Equation (8). Let us rewrite this solution as the wave function

$$
\psi_{0}^{n}(z, t)=\left(\frac{1}{\sqrt{\pi} n ! 2^{n}}\right)^{1 / 2} H_{n}(z) \exp \left\{-\left[\frac{z^{2}+t^{2}}{2}\right]\right\} .
$$

The subscript 0 means that the wave function is for the hadron at rest. The above expression is not Lorentz-invariant, and its localization undergoes a Lorentz squeeze as the hadron moves along the $z$ direction as shown in Figure 4.

For this Lorentz-covariant system, it is convenient to use the light-cone variables

$$
u=\frac{z+t}{\sqrt{2}}, \quad v=\frac{z-t}{\sqrt{2}}
$$


introduced in Equation (12). The lorentz-boost along the $z$ axis leads to

$$
u \rightarrow u^{\prime}=\mathrm{e}^{\eta} u, \quad v \rightarrow v^{\prime}=\mathrm{e}^{-\eta} v,
$$

where $\eta$ is the boost parameter and is $\tanh \eta=v / c$. In terms of these lightcone variables, the wave function of Equation (40) can be written as

$$
\psi_{0}^{n}(x, t)=\left(\frac{1}{\pi n ! 2^{2}}\right)^{1 / 2} H_{n}\left(\frac{u+v}{\sqrt{2}}\right) \exp \left(-\left[\frac{u^{2}+v^{2}}{2}\right]\right) .
$$

If the system is boosted, the wave function becomes

$$
\psi_{\eta}^{n}(x, t)=\left(\frac{1}{\pi n ! 2^{2}}\right)^{1 / 2} H_{n}\left(\frac{\mathrm{e}^{-\eta} u+\mathrm{e}^{\eta} v}{\sqrt{2}}\right) \exp \left(-\left[\frac{\mathrm{e}^{-2 \eta} u^{2}+\mathrm{e}^{2 \eta} v^{2}}{2}\right]\right) .
$$

This wave function can be expanded as [24]

$$
\psi_{\eta}^{n}(z, t)=\left(\frac{1}{\cosh \eta}\right)^{(n+1)} \sum_{k}\left[\frac{(n+k) !}{n ! k !}\right]^{1 / 2}(\tanh \eta)^{k} \chi_{n+k}(z) \chi_{k}(t),
$$

where $\chi_{n}(z)$ is the $n$-th excited-state oscillator wave function.

Here comes the fundamental problem. If the hadron is at rest, this wave function is separable in $z$ and $t$. If the $t$ variable is integrated out, the rest is the present form of non-relativistic quantum mechanics.

However, if the hadron moves and gains speed, the $t$ dependence becomes non-separable, and we have to resort to density matrix. From the wave function of Equation (6), we can construct the pure-state density matrix

$$
\rho_{\eta}^{n}\left(z, t ; z^{\prime}, t^{\prime}\right)=\psi_{\eta}^{n}(z, t)\left[\psi_{\eta}^{n}\left(z^{\prime}, t^{\prime}\right)\right]^{*},
$$

where $\left[\psi_{\eta}^{n}\left(z^{\prime}, t^{\prime}\right)\right]^{*}=\psi_{\eta}^{n}\left(z^{\prime}, t^{\prime}\right)$. This pure-state density matrix satisfies the condition $\rho^{2}=\rho$ :

$$
\rho_{\eta}\left(z, t ; x^{\prime}, t^{\prime}\right)=\int \rho_{\eta}\left(z, t ; x^{\prime \prime}, t^{\prime \prime}\right) \rho_{\eta}\left(z^{\prime \prime}, t^{\prime \prime} ; z^{\prime}, t^{\prime}\right) \mathrm{d} z^{\prime \prime} \mathrm{d} t^{\prime \prime} .
$$

However, there are at present no measurement theories which accommodate the time-separation variable $t$. Thus, we can take the trace of the $\rho$ matrix with respect to the $t$ variable. Then the resulting density matrix is

$$
\begin{gathered}
\rho_{\eta}^{n}\left(z, z^{\prime}\right)=\int \psi_{\eta}^{n}(z, t) \psi_{\eta}^{n}\left(z^{\prime}, t\right) \mathrm{d} t \\
=\left(\frac{1}{\cosh \eta}\right)^{2(n+1)} \sum_{k} \frac{(n+k) !}{n ! k !}(\tanh \eta)^{2 k} \chi_{n+k}(z) \chi_{n+k}\left(z^{\prime}\right) .
\end{gathered}
$$

The trace of this density matrix is one, but the trace of $\rho^{2}$ is less than one, as

$$
\begin{gathered}
\operatorname{Tr}\left(\rho^{2}\right)=\int \rho\left(z, z^{\prime}\right) \rho\left(z^{\prime}, z\right) \mathrm{d} z^{\prime} \mathrm{d} z \\
=\left(\frac{1}{\cosh \eta}\right)^{4(n+1)} \sum_{k}\left[\frac{(n+k) !}{n ! k !}\right]^{2 k}(\tanh \eta)^{4 k} .
\end{gathered}
$$

which is less than one. This is due to the fact that we do not know how to deal with the time-like separation in the present formulation of quantum mechanics. Our knowledge is less than complete. 
The standard way to measure this ignorance is to calculate the entropy defined as [1] [2] [3] [27]

$$
S=-\operatorname{Tr}[\rho \ln (\rho)] .
$$

This formula is known as the Shannon entropy in the current literature on quantum computation and quantum information [11].

If we pretend to know the distribution along the time-like direction and use the pure-state density matrix given in Equation (46), then the entropy is zero. However, if we do not know how to deal with the distribution along the time separation $t$, then we should use the density matrix of Equation (48) to calculate the entropy, and the result is [27]

$$
\begin{aligned}
& S=2(n+1)\left[(\cosh \eta)^{2} \ln (\cosh \eta)-(\sinh \eta)^{2} \ln (\sinh \eta)\right] \\
& -\left(\frac{1}{\cosh \eta}\right)^{2(n+1)} \sum_{k} \frac{(n+k) !}{n ! k !}\left[\ln \left[\frac{(n+k) !}{n ! k !}\right]\right](\tanh \eta)^{2 k} .
\end{aligned}
$$

Let us go back to the wave function given in Equation (6). As is illustrated in Figure 4, its localization property is dictated by the Gaussian factor which corresponds to the ground-state wave function. For this reason, we expect that much of the behavior of the density matrix or the entropy for the $n$-th excited state will be the same as that for the ground state with $n=0$. For this state, the density matrix and the entropy are

$$
\rho\left(z, z^{\prime}\right)=\left(\frac{1}{\pi \cosh (2 \eta)}\right)^{1 / 2} \exp \left\{-\frac{1}{4}\left[\frac{\left(z+z^{\prime}\right)^{2}}{\cosh (2 \eta)}-\left(z-z^{\prime}\right)^{2} \cosh (2 \eta)\right]\right\},
$$

and

$$
S=\left(\cosh ^{2} \eta\right) \ln \left(\cosh ^{2} \eta\right)-\left(\sinh ^{2} \eta\right) \ln \left(\sinh ^{2} \eta\right),
$$

respectively. The quark distribution $\rho(z, z)$ becomes

$$
\rho(z, z)=\left[\frac{1}{\pi \cosh (2 \eta)}\right]^{1 / 2} \exp \left(\frac{-z^{2}}{\cosh (2 \eta)}\right) .
$$

The width of the distribution becomes $\cosh (2 \eta)$, and becomes wide-spread as the hadronic speed increases. Likewise, the momentum distribution becomes wide-spread, as in the case of Feynman's parton picture described in Subsection 5.1. This simultaneous increase in the momentum and position distribution widths is called the parton phenomenon in high-energy physics [13] [14] [15]. The position-momentum uncertainty becomes $\cosh ^{2} \eta$. This increase in uncertainty is due to our ignorance about the physical but unmeasurable time-separation variable. This does not violate the fundamental law of the uncertainty as described in Subsection 5.2.

The use of an unmeasurable variable as a shadow coordinate is not new in physics [7] [8]. Feynman's book on statistical mechanics contains the following paragraph [5]. 
When we solve a quantum-mechanical problem, what we really do is divide the universe into two parts-the system in which we are interested and the rest of the universe. We then usually act as if the system in which we are interested comprised the entire universe. To motivate the use of density matrices, let us see what happens when we include the part of the universe outside the system.

In the present paper, we have identified Feynman's rest of the universe as the time-separation coordinate in a relativistic two-body problem. Our ignorance about this coordinate leads to a density matrix for a non-pure state, and consequently to an increase of entropy. Figure 9 shows the entropy as a function of the hadronic speed or $\tan \eta$. The entropy is zero when the hadron is at rest. It increases rapidly as the hadronic speed approaches the speed of light.

Finally, let us examine how the ignorance will lead to the concept of temperature [28]. For the Lorentz-boosted ground state, the density matrix of Equation (48) becomes

$$
\rho_{\eta}\left(z, z^{\prime}\right)=\left(\frac{1}{\cosh \eta}\right) \sum_{k}(\tanh \eta)^{2 k} \chi_{k}(z) \chi_{k}\left(z^{\prime}\right) .
$$

We can now compare this expression for the oscillator system in the thermally excited state. In terms of the temperature $T$, the density matrix takes the form [46] [47].

$$
\rho_{T}\left(z, z^{\prime}\right)=\left(1-\mathrm{e}^{-1 / T}\right) \sum_{n} \mathrm{e}^{-n / T} \chi_{n}(z) \chi_{n}\left(z^{\prime}\right),
$$

where $T$ means $k T / \hbar \omega$, with $k$ and $\omega$ as Boltzmann's constant and the frequency of oscillation respectively. If we compare this expression with Equation (58). Then

$$
\mathrm{e}^{-1 / T}=(\tanh \eta)^{2}
$$

This leads to

$$
T=\frac{-1}{2 \ln (\tanh \eta)}
$$
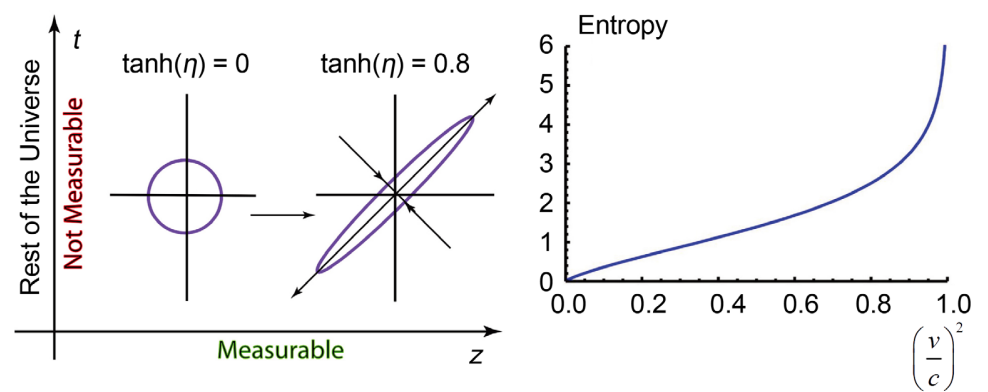

Figure 9. Feynman's rest of the universe and entropy. For the hadron, the space-like extension is measurable, but the time-like separation is not. It is in the rest of the universe. This non-measurable variable should be treated statistically. It leads to the increase in entropy. 
The temperature rises rapidly as the hadronic speed approaches the speed of light, as indicated in Figure 8. As we noted in Subsection 5.1, the hadron becomes a plasma state as in the case of Feynman's parton picture.

\section{Concluding Remarks}

The primary purpose of this paper was to study the entropy and temperature of bound states in the Lorentz-covariant world. The result of study is given in Section 6.

This problem arises because the time separation between the constituent particles is not a measurable quantity in the present form of quantum mechanics. On the other hand, it is possible to treat this unmeasurable variable statistically. The time separation is negligible when the bound state is at rest, but it becomes as significant as the space separation (like the Bohr radius) when the bound state moves.

In order to study this effect, we need at least one Lorentz-covariant model for bound states. Dirac and Feynman made their efforts to construct such a wave function. Much of the present paper is devoted to the integration of their efforts to construct wave functions for moving bound states in Einstein's Lorentzcovariant world.

It was Paul A. M. Dirac who made efforts to construct wave functions for moving bound states. Dirac's papers and books are like beautiful poems, but they do not contain figures. It was a challenge to convert his poems [18] [19] [20] into the circle and the rectangle given in Figure 4. Then it is easy to integrate those two figures. A more detailed explanation is given in a recent book entitled Physics of the Lorentz Group, 2nd Edition by Başkal, Kim, and Noz [24].

We used in this paper the Lorentz-covariant wave function which provides the resolution to the question whether the quarks and partons are two different ways of looking at the same entity in the Lorentz-covariant world, as illustrated in Figure 8. This also provides the answer to the Bohr-Einstein issue of moving hydrogen atoms.

\section{Acknowledgements}

This review paper is largely based on the papers and books I, the author of this paper, published with Marilyn Noz since 1973. I thank her for this prolonged collaboration. I thank also my younger colleagues who co-authored with me many papers I published since 1978. Their names are D. Han, P. Hussar, S. H. Oh, and D. Son. My later co-authors include S. Başkal, and E. Georgieva.

In July of 1962, I became an assistant professor of physics at the University of Maryland. Paul A. M. Dirac visited the University for one week in October of the same year at the invitation of John S. Tall who was the chairman of the physics department. Toll assigned me as the personal assistant to Dirac. This gave me an excellent opportunity to learn physics directly from Dirac.

Dirac told me that physicists in general do not understand the difference be- 
tween the Lorentz invariance and the Lorentz covariance. This difference was illustrated in terms of the hyperbola and the squeezed circle Figure 4 . This allowed me to integrate Dirac's attempts to construct the wave function in the Lorentz-covariant world.

Another fundamental issue is the unification of the internal space-time symmetries. In his paper of 1939 [25], he stated that those symmetries are like $O(3)$ and and $E(2)$ respectively. The question then is whether the $E(2)$ symmetry can be obtained continuously as the mass of particle becomes zero. This question has a stormy history [48]. Because the transition from $O(3)$ to $E(2)$ is not an analytic continuation. I am grateful to Professor Eugene Paul Wigner for spending time with me to settle this issue as described in Section 2 of the present paper.

\section{Conflicts of Interest}

The author declares no conflicts of interest regarding the publication of this paper.

\section{References}

[1] von Neumann, J. (1932) Die mathematische Grundlagen der Quanten-mechanik. Springer, Berlin. (See also von Neumann, I. (1955) Mathematical Foundation of Quantum Mechanics. Princeton University Press, Princeton.)

[2] Fano, U. (1957) Reviews of Modern Physics, 29, 74-93. https://doi.org/10.1103/RevModPhys.29.74

[3] Wigner, E.P. and Yanase, M.M. (1963) Proceedings of the National Academy of Sciences of the United States of America, 49, 910-918. https://doi.org/10.1073/pnas.49.6.910

[4] Fetter, A.L. and Walecka, J.D. (1971) Quantum Theory of Many-Particle Systems. McGraw-Hill, New York.

[5] Feynman, R. P. (1972) Statistical Mechanics. Benjamin Cummings, Reading.

[6] Blum, K. (1981) Density Matrix Theory and Applications. Plenum Press, New York and London. https://doi.org/10.1007/978-1-4615-6808-7

[7] Umezawa, H., Matsumoto, H. and Tachiki, M. (1982) Thermo Field Dynamics and Condensed States. North-Holland, Amsterdam.

[8] Mann; A. and Revzen, M. (1989) Physics Letters A, 134, 273-275. https://doi.org/10.1016/0375-9601(89)90635-X

[9] Ekert, A.K. and Knight, P.L. (1989) American Journal of Physics, 57, 692-697. https://doi.org/10.1119/1.15922

[10] Barnett, S.M. and Phoenix, S.J.D. (1991) Physical Review A, 44, 535-545. https://doi.org/10.1103/PhysRevA.44.535

[11] Nelson, M.A. and Chuang, I.L. (2016) Quantum Computation and Quantum Information. Cambridge University Press, Cambridge.

[12] Gell-Mann, M. (1964) Physics Letters, 8, 214-215. https://doi.org/10.1016/S0031-9163(64)92001-3

[13] Feynman, R.P. (1969) Physical Review Letters, 23, 1415-1417. https://doi.org/10.1103/PhysRevLett.23.1415

[14] Feynman, R.P. (1969) The Behavior of Hadron Collisions at Extreme Energies. In: 
Yang, C.N., et al., Eds., High-Energy Collisions: Proceedings of the Third International Conference, Gordon and Breach, New York, 237-249.

[15] Bjorken, J.D. and Paschos, E.A. (1969) Physical Review, 185, 1975-1982. https://doi.org/10.1103/PhysRev.185.1975

[16] Kim, Y.S. and Noz, M.E. (1977) Physical Review D, 15, 335-338. https://doi.org/10.1103/PhysRevD.15.335

[17] Kim, Y.S. (1989) Physical Review Letters, 63, 348-351. https://doi.org/10.1103/PhysRevLett.63.348

[18] Dirac, P.A.M. (1927) Proceedings of the Royal Society of London. Series A, Containing Papers of a Mathematical and Physical Character, 114, 243-265. https://doi.org/10.1098/rspa.1927.0039

[19] Dirac, P.A.M. (1945) Proceedings of the Royal Society of London. Series A, Containing Papers of a Mathematical and Physical Character, 183, 284-295. https://doi.org/10.1098/rspa.1945.0003

[20] Dirac, P.A.M. (1949) Reviews of Modern Physics, 21, 392-399. https://doi.org/10.1103/RevModPhys.21.392

[21] Dirac, P. A. M. (1963) Journal of Mathematical Physics, 4, 901-909. https://doi.org/10.1063/1.1704016

[22] Kim, Y.S., Noz, M.E. and Oh, S.H. (1979) Journal of Mathematical Physics, 20, 1341 1344. https://doi.org/10.1063/1.524237

[23] Kim, Y.S. and Noz, M.E. (2020) Symmetry, 12, Article No. 1270. https://doi.org/10.3390/sym12081270

[24] Baskal, S., Kim, Y.S. and Noz, M.E. (2021) Physics of the Lorentz Group (2nd Edition): Beyond High-Energy Physics and Optics. IOP Publishing, Bristol. https://doi.org/10.1088/978-0-7503-3607-9

[25] Wigner, E. (1939) Annals of Mathematics, 40, 149-204.

[26] Feynman, R.P., Kislinger, M. and Ravndal, F. (1971) Physical Review D, 3, 27062732. https://doi.org/10.1103/PhysRevD.3.2706

[27] Kim, Y.S. and Wigner, E.P. (1990) Physics Letters A, 147, 343-347. https://doi.org/10.1016/0375-9601(90)90550-8

[28] Han, D., Kim, Y.S. and Noz, M.E. (1990) Physics Letters A, 144, 111-115. https://doi.org/10.1016/0375-9601(90)90684-G

[29] Kim, Y.S. and Wigner, E.P. (1987) Journal of Mathematical Physics, 28, 1175-1179. https://doi.org/10.1063/1.527824

[30] Kim, Y.S. and Wigner, E.P. (1990) Journal of Mathematical Physics, 31, 55-60. https://doi.org/10.1063/1.528827

[31] Başkal, S., Kim, Y.S. and Noz, M. E. (2019) Symmetry, 11, Article No. 409. https://doi.org/10.3390/sym11030409

[32] Başkal, S, Kim, Y.S. and Noz, M.E. (2019) Quantum Reports, 1, 236-251. https://doi.org/10.3390/quantum1020021

[33] Wigner, E. P. (1972) On the Time-Energy Uncertainty Relation. In: Salam, A. and Wigner, E. P., Eds. Aspects of Quantum Theory, Cambridge University Press, London, 237-247.

[34] Fujimura, K., Kobayashi, T. and Namiki, M. (1970) Progress of Theoretical Physics, 43, 73-79.

[35] Beg, M.A.B., Lee, B.W. and Pais, A. (1964) Physical Review Letters, 13, 514-517. https://doi.org/10.1103/PhysRevLett.13.514 
[36] Greenberg, O.W. and Resnikoff, M. (1967) Physical Review, 163, 1844-1851. https://doi.org/10.1103/PhysRev.163.1844

[37] Yukawa, H. (1953) Physical Review, 91, 415-416.

https://doi.org/10.1103/PhysRev.91.415.2

[38] Markov, M. (1956) Il Nuovo Cimento, 3, 760-772. https://doi.org/10.1007/BF02746074

[39] Robert Hofstadter, R. (1956) Reviews of Modern Physics, 28, 214-254. https://doi.org/10.1103/RevModPhys.28.214

[40] Hofstadter, R. and McAllister, R.W. (1955) Physical Review, 98, 217-218. https://doi.org/10.1103/PhysRev.98.217

[41] Ginzburg, V.L. and Man'ko, V.I. (1965) Nuclear Physics, 74, 577-588. https://doi.org/10.1016/0029-5582(65)90203-8

[42] Licht, A.L. and Pagnamenta, A. (1970) Physical Review D, 2, 1150-1156. https://doi.org/10.1103/PhysRevD.2.1150

[43] Lipes, R. (1972) Physical Review D, 5, 2849-2863. https://doi.org/10.1103/PhysRevD.5.2849

[44] Kim, Y.S. and Noz, M.E. (1973) Physical Review D, 8, 3521-3627. https://doi.org/10.1103/PhysRevD.8.3521

[45] Henriques, A.B., Keller, B.H. and Moorhouse, R.G. (1975) Annals of Physics, 93, 125-151. https://doi.org/10.1016/0003-4916(75)90209-2

[46] Landau, L.D. and Lifshitz, E.M. (1958) Statistical Physics. Pergamon Press, London.

[47] Davies, R.W. and Davies, K.T.R. (1975) Annals of Physics, 89, 261-273. https://doi.org/10.1016/0003-4916(75)90182-7

[48] Saar, R. and Groote. S. (2017) Advances in Applied Clifford Algebras, 27, 2739-2768. https://doi.org/10.1007/s00006-017-0758-2

[49] Ruiz, M.J. (1974) Physical Review D, 10, 4306-4307. https://doi.org/10.1103/PhysRevD.10.4306 


\section{Appendix}

In Section 4, we noted that the bound state has its internal space-time coordinates, and we considered a bound state equation in Equation (26). Let us write this oscillator equation:

$$
\frac{1}{2}\left(-\left[\frac{\partial}{\partial x_{\mu}}\right]^{2}+x_{\mu}^{2}\right) \psi(x)=\lambda \psi(x) .
$$

This differential equation is separable in all space-time variables. Thus we can concentrate on the longitudinal and time coordinates. The equation then becomes

$$
\frac{1}{2}\left[\left(-\left[\frac{\partial}{\partial z}\right]^{2}+z^{2}\right)-\left(-\left[\frac{\partial}{\partial t}\right]^{2}+t^{2}\right)\right] \psi(z, t)=\lambda \psi(z, t) .
$$

Since the time excitations are not allowed, the solution of this equation takes the form

$$
\psi^{n}(z, t)=\left(\frac{1}{\pi}\right)^{1 / 4} \exp \left(\frac{-t^{2}}{2}\right) \xi_{n}(z)
$$

where $\xi_{n}(z)$ is the oscillator wave function for the $\mathrm{n}$-th excited state. The differential equation of Equation (63) is invariant under the Lorentz boost:

$$
z \rightarrow \frac{z+\beta t}{\sqrt{1-\beta^{2}}}, \quad t \rightarrow \frac{t+\beta z}{\sqrt{1-\beta^{2}}},
$$

and the boosted wave function becomes

$$
\psi_{\beta}^{n}(z, t)=\left(\frac{1}{\pi}\right)^{1 / 4} \exp \left(\frac{-t^{\prime 2}}{2}\right) \phi_{n}\left(z^{\prime}\right),
$$

with

$$
z^{\prime}=\frac{z-\beta t}{\sqrt{1-\beta^{2}}}, \quad t^{\prime}=\frac{t-\beta z}{\sqrt{1-\beta^{2}}} .
$$

The wave function of Equation (66) becomes $\psi^{n}(z, t)$ given in Equation (64) when $\beta=0$. There are no excitations along the $t$ direction because of the $c$ number time-energy uncertainty relation [18].

It is then of interest to evaluate the integral [24] [49], and the result is

$$
\int \psi^{n}(z, t) \psi_{\beta}^{n^{\prime}}(z, t) \mathrm{d} x \mathrm{~d} t=\left(\sqrt{1-\beta^{2}}\right)^{(n+1)} \delta_{n n^{\prime}} .
$$

The orthogonality relation and the contraction property contained in this formula are illustrated in Figure A1. The stationary ground state wave function is orthogonal to all excited states. This ground state is contracted by $\sqrt{1-\beta^{2}}$. This is consistent with our understanding of Einstein's Lorentz contraction of a rod.

Then why is $\left[\sqrt{1-\beta^{2}}\right]^{(n+1)}$ for the $n$-th excited states? It is because the (wavefunction $)^{2}$ has $(n+1)$ humps. Then why are they multiplicative? 


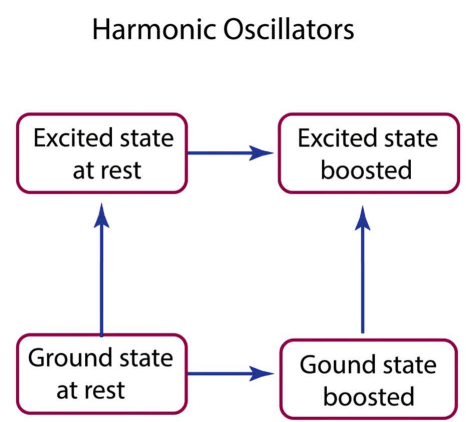

(a)

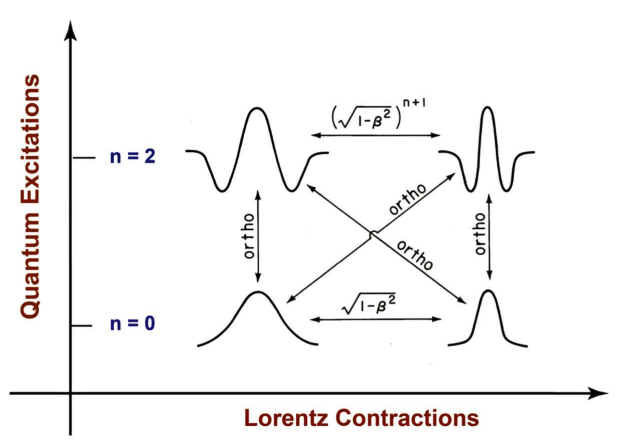

(b)

Figure A1. Orthogonality and Lorentz contractions of the covariant harmonic wave functions, with words for (a) and graphs for (b). The orthogonality is maintained for all excites sates. The Lorentzcontraction factor of $\sqrt{1-\beta^{2}}$ for the ground state is consistent with Einstein's Lorentz contraction. For the $n$-th excited state, the $\mid$ wavefunction $\left.\right|^{2}$ has $(1+n)$ humps. Thus the net contraction thus should $\left[\sqrt{1-\beta^{2}}\right]^{(n+1)}$.

In order to answer this question, let us use the bra-and-ket notation for the harmonic oscillators, where the ground state is $|0\rangle$ and $|n\rangle$ is for the $\mathrm{n}$-th excited state. We use $a$ and $a^{\dagger}$ for step-down and step-up operators respectively. As is well known, the $\mathrm{n}$-th excited state becomes

$$
|n\rangle=\frac{1}{\sqrt{n !}}\left(a^{\dagger}\right)^{n}|0\rangle .
$$

Thus, each additional hump is produced through the multiplication process. 